\title{
Back-Stepping Fuzzy Adaptive Sliding Mode Trajectory Tracking Control for Wall-Climbing Robot
}

\author{
Lai Xin ${ }^{1 *}$, Liu Pengli ${ }^{1}$, Lu Yang ${ }^{2}$, Wang Chunpu ${ }^{3}$ \\ ${ }^{1}$ Key Laboratory of Oil \& Gas Equipment of Education Ministry, Southwest Petroleum University, Sichuan, \\ 610500 , China. \\ 2Deyang College of Urban Rail Transit, Deyang, 618400, China. \\ ${ }^{3}$ Chuanqing Geological Exploration and Development Research Institute, 610051, China. \\ * Corresponding author. Email: xinxinluck107@163.com \\ Manuscript submitted August 19, 2019; accepted October 8, 2019. \\ doi: 10.17706/jcp.14.12.662-679
}

\begin{abstract}
To improve the robustness and control precision of trajectory tracking for the four-wheel-driven wall-climbing robot, a novel back-stepping adaptive fuzzy sliding mode control (BFASMC) is proposed in the paper. Firstly, in order to make the tracking error between the actual trajectory and the reference trajectory of the WCR approach zero, the kinematic controller is designed based on the back-stepping method. Then, the output of the kinematic controller is used as the auxiliary speed input of the dynamic controller. To reduce chattering and restrain the uncertain parameters of the WCR, an adaptive control and a fuzzy control are introduced into a back-stepping sliding mode control (BSMC) to form the proposed method. Finally, simulation is conducted to track the straight line and circle. The results show that the proposed method improves the control precision and stability, and reduces the chattering in the process of switching sliding surfaces effectively.
\end{abstract}

Key words: Wall-climbing robot, back-stepping, adaptive control, fuzzy control, sliding mode control.

\section{Introduction}

Wall-climbing robot (WCR) is very important in detecting defects in storage tanks and oil pipelines for the petrochemical industry. The most commonly used wall-climbing robot is divided into wheel, foot and crawler [1], [2]. Considerable research and application have been utilized because of its relatively simple structure and movement, such as the permanent magnet adsorption omnidirectional wheel-driven wall-climbing robot developed [3]. It can adapt to various surface curvatures and it has better mobility than previous models.

The wall-climbing robot is a type of nonholonomic mobile device influenced by nonlinear and strong coupling [4]-[6]. Trajectory tracking control is an important part of the control system of the wheeled climbing robot [7], [8]. The kinematics model is basically the same as the ground-wheel-climbing robot [9]. It is important that the dynamic model of the wheeled wall-climbing robot allows for the influence of adsorption force and gravity [10]. Therefore, in order to solve the problem of trajectory tracking in the wheeled wall-climbing robot, it is necessary to consider the influence of the force and instability factors to establish a complete dynamic model [11]-[13].

The kinetic model of the wheeled-climbing-wall robot was based on the momentum and the kinetic energy theories of variable mass Newtonian mechanics, which considered the influence of carrying the cable quality on the motion performance of the robot, but did not perform a stress analysis for friction [14]. The sliding 
mode variable structure control is an important nonlinear control method with the advantages of being unaffected by parameter change and disturbances, quick to respond and without need for system on-line identification [15]-[18]. It is widely used in the field of robot control; however, the inevitable chattering effect that occurs in the process of a switching sliding surface influences the control performance of the system [19]. [20] proposed an adaptive sliding-mode controller based on the "Super-Twist" state observer (ASMC), the ASMC can provide a highly efficient control of nonlinear systems in the presence of nonmodeled dynamics and external perturbations. [21] proposed a sliding mode adaptive neural network tracking controller (SMANNC), the SMANNC can achieve a stable closed-loop system for the trajectory-tracking control of a mobile robot with unknown nonlinear dynamics. [22] proposed a novel adaptive dynamic sliding mode controller (ADSMC) with integrator in control loop for the trajectory tracking, for compensating uncertainties and disturbances, the equivalent control is augmented by a discontinuous control. [23] proposed a fuzzy adaptive multi-mode sliding mode control (FAMMSMC) for precision linear stage. Although the FAMSMC method can effectively reduce chattering, the control accuracy is relatively low and the pose error is relatively large. [24] proposed a back-stepping adaptive sliding mode (BASMC) trajectory tracking control for a quadrotor. The BASMC simplifies the controller design by using intermediate virtual control variables and Lyapunov functions, but fails to suppress chattering effectively. [25] proposed a back-stepping sliding mode control (BSMC) for variable speed wind turbine, but it still doesn't solve the chattering problem.

To address the chattering and the uncertain parameters of the WCR, we propose a new trajectory tracking control in this paper. Our main contributions are summarized as follows:

1. To improve the precision and stability of trajectory tracking for the WCR, the novel control method is proposed in the paper to effectively reduce the chattering effect in the process of switching sliding surfaces.

2. In this paper, a new back-stepping fuzzy adaptive sliding mode controller (BFASMC) is proposed. Based on the kinematic and dynamic model of the WCR, the kinematic controller and dynamic controller of the WCR are designed. The control method with global asymptotic stability is proposed, and the stability of the system is proved by the theory of Lyapunov.

3. The kinematic controller of the WCR is obtained based back-stepping method. To the best of our knowledge, this is the first time to use the output of the kinematic controller is used as the auxiliary speed input of the dynamics controller.

4. The experiments show that the designed controller has tracked the straight line and circular reference trajectories of the WCR, and the proposed BFASMC method outperform the back-stepping adaptive sliding mode controller (BASMC). The simulation results demonstrate our proposed method is robust and real-time in the motion control of the wall-climbing robot.

The novel BFASMC tracking structure is shown in Fig. 1. Firstly, the control function based on back-stepping is substituted into the differential equation $\left(q_{e}\right)$ of the WCR pose error to design kinematic controller. Secondly, the dynamic controller of the WCR is designed by sliding mode control. Then the auxiliary velocity ( $\dot{q}_{e}$ ) of the output of the kinematics controller is selected as the input of the dynamics controller. At the same time, the gain $(\varepsilon)$ of the sliding mode control is adjusted by the fuzzy control principle, and the uncertain parameters $\left(\tau_{d}\right)$ of the dynamic model are estimated by the adaptive method. Next, the dynamic equation is derived from the torque $(\tau)$ output of the dynamic controller. Finally, according to the velocity $(v)$ of the output of the kinetic equation, the kinematics equation is obtained. The actual pose $(q)$ of the WCR is compared with the reference pose $\left(q_{r}\right)$, hence the pose $\operatorname{error}\left(q_{e}\right)$ is gain. So far, the feedback closed-loop control system of the WCR has been completely designed. The control system can greatly reduce the 
chattering and steady-state error, and it makes the WCR complete trajectory tracking and reaches a stable state in a relatively short time.

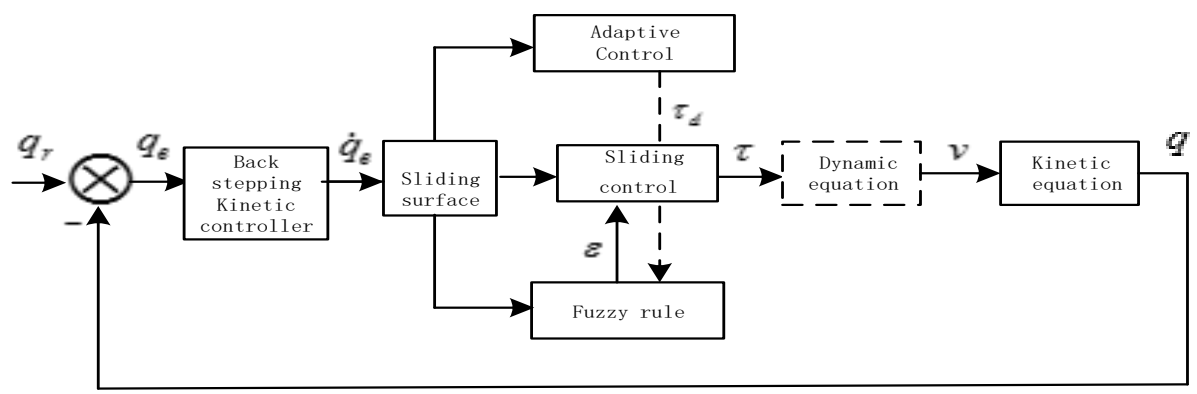

Fig. 1. Structure of BAFSMC of the WCR.

\section{Kinematic Analysis of Wall-Climbing Robot}

\subsection{Mathematical Model of Wall-Climbing Robot}

The wheel-climbing robot is a variety of the typical nonholonomic robot, and it is also a complicated nonlinear system [26]. A typical four-wheeled mobile robot geometry model is shown in Fig. 2. The center of mass $(M)$ and geometric center $(C)$ do not coincide and the spacing is $l$. The mobile robot is powered by four driving wheels with a diameter of $r$. The relative distance between the two wheels is $2 b$, and the distance between the adjacent wheels is $2 d . \theta$ is the direction angle, which is the attitude angle of the robot movement; $\dot{x}$ and $y$ are respectively the speed of the robot along the $X$ axis direction and $Y$ axis direction in Cartesian coordinates.

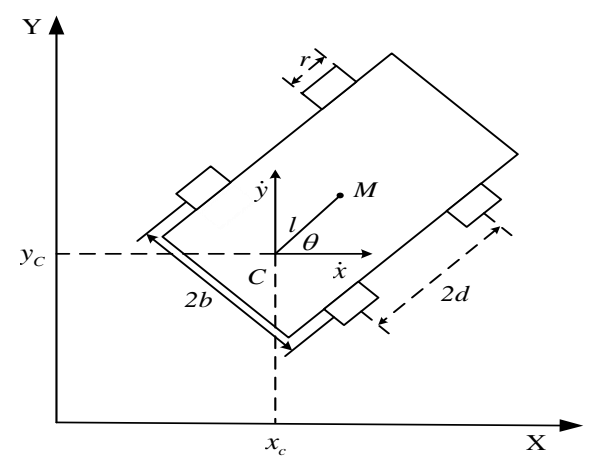

Fig. 2. Schematic diagram of four-wheel drive mobile robot.

To meet the non-sliding conditions and improve the reliability of the mobile robot, the following assumptions are made before analyzing the constraint equations of the four-wheel drive wall-climbing robot.

The motion plane of the wall-climbing robot is a horizontal plane. The mass distribution of the car body is even, and the geometric center and mass center of the wall-climbing robot coincide at this time.

\subsection{Kinematic Controller Based Back-Stepping}

According to the back-stepping method, $\bar{\theta}_{e}$ [27] is selected,

$$
\bar{\theta}_{e}=\theta_{e}+\arcsin \left(\frac{k(t) y_{e}}{\sqrt{1+x_{e}^{2}+y_{e}^{2}}}\right)
$$


where $x_{e}, y_{e}$ and $\theta_{e}$ are respectively the pose errors of WCR along horizontal direction, vertical direction and steering angle, and $\bar{\theta}_{e}$ is the estimated value of $\theta_{e} \cdot k(t)=\lambda_{1} v_{r}+\lambda_{2} \sin (t), \lambda_{1}$ and $\lambda_{2}$ are the positive constants, its constraint inequality is: $\lambda_{1}\left|v_{r}\right|_{\max }+\lambda_{2} \leq 0.5,\left|v_{r}\right|_{\max }$ is the maximum value of $\left|v_{r}\right|$. This constraint can make $k(t)$ less than or equal to 0.5 for any value of $t$. By substituting equation (1) into the differential equation of position error of wall-climbing robot, the following equation can be obtained,

$$
\left[\begin{array}{c}
\dot{x}_{e} \\
\dot{y}_{e} \\
\dot{\theta}_{e}
\end{array}\right]=\left[\begin{array}{c}
\omega y_{e}+v+g_{1} \\
-\omega x_{e}-\frac{k(t) v_{r} y_{e}}{\Phi_{1}}+g_{2} \\
\omega\left(1-\frac{k(t) x_{e}}{\Phi_{2}}\right)+g_{3}-v \frac{k(t) x_{e} y_{e}}{\Phi_{1}^{2} \Phi_{2}}
\end{array} \mid\right.
$$

where,

$$
\begin{aligned}
& \Phi_{1}=\sqrt{1+x_{e}^{2}+y_{e}^{2}}, \quad \Phi_{2}=\sqrt{1+x_{e}^{2}+\left(1-k^{2}(t)\right) y_{e}^{2}}, \quad g_{1}=-\frac{v_{r} \cos \bar{\theta}_{e} \Phi_{2}}{\Phi_{1}}-\frac{v_{r} k(t) y_{e} \sin \bar{\theta}_{e}}{\Phi_{1}}, \\
& g_{2}=\frac{v_{r}\left(\cos \bar{\theta}_{e}-1\right) \Phi_{2}}{\Phi_{1}}-\frac{v_{r} k(t) y_{e} \sin \bar{\theta}_{e}}{\Phi_{2}}, g_{3}=-\omega_{r}+\frac{\dot{k}(t) y_{e}+k(t) g_{2}-\frac{k(t)\left(x_{e} g_{1}+y_{e} g_{2}\right)}{\Phi_{1}^{2}}}{\Phi_{2}} .
\end{aligned}
$$

Let $v_{c}$ and $\omega_{c}$ be the auxiliary speed control of $v$ and $\omega, \tilde{v}$ and $\tilde{\omega}$ be the auxiliary speed control error, then,

$$
\dot{q}_{e}=\left[\begin{array}{c}
\tilde{v}=v-v_{c}, \tilde{\omega}=\omega-\omega_{c} \\
\left(\omega_{c}+\tilde{\omega}\right) y_{e}+v_{c}+\tilde{v}+g_{1} \\
-\left(\omega_{c}+\tilde{\omega}\right) x_{e}-\frac{k(t) v_{r} y_{e}}{\Phi_{1}}+g_{2} \\
\left(\omega_{c}+\tilde{\omega}\right)\left(1-\frac{k(t) x_{e}}{\Phi_{1}}\right)+g_{3}-\left(v_{c}+\tilde{v}\right) \frac{k(t) x_{e} y_{e}}{\Phi_{1}^{2} \Phi_{2}}
\end{array} \mid\right.
$$

The following Lyapunov function is chosen as the stability criterion,

$$
V_{1}=\sqrt{1+x_{e}^{2}+y_{e}^{2}}+\sqrt{1+\bar{\theta}_{e}^{2}}
$$

The time derivative of $V_{1}$ can be obtained,

$$
\begin{aligned}
\dot{V}_{1}= & \left.-\frac{k(t) v_{r} y_{e}^{2}}{\Phi_{1}}+\frac{x_{e}}{\Phi_{1}}\left(v_{c}+g_{1}\right)+\frac{y_{e} g_{2}}{\Phi_{1}}+\frac{\bar{\theta}_{e}}{\Phi_{3}} \mid \omega_{c}\left(1-\frac{k(t) x_{e}}{\Phi_{1}}\right)+g_{3}-v_{c} \frac{k(t) x_{e} y_{e}}{\Phi_{1}^{2} \Phi_{2}}\right] \\
& +\tilde{v}\left(\frac{x_{e}}{\Phi_{1}}-\frac{k(t) x_{e} y_{e} \bar{\theta}_{e}}{\Phi_{1}^{2} \Phi_{2} \Phi_{3}}\right)+\tilde{\omega} \frac{\bar{\theta}_{e}}{\Phi_{3}}\left(1-\frac{k(t) x_{e}}{\Phi_{1}}\right)
\end{aligned}
$$

where $\Phi_{3}=\sqrt{1+\bar{\theta}_{e}^{2}}$. The auxiliary velocity of the WCR is selected as, 


$$
v_{c}=-k_{1} \frac{x_{e}}{\Phi_{1}}-g_{1}, \quad \omega_{c}=\frac{1}{\left(1-\frac{k(t) x_{e}}{\Phi_{1}}\right)}\left(-k_{2} \bar{\theta}_{e}-g_{3}+v_{c} \frac{k(t) x_{e} y_{e}}{\Phi_{1}^{2} \Phi_{2}}\right)
$$

The kinematic controller Lyapunov function can be further rewritten as:

$$
\dot{V}_{1}=-K_{1} \frac{x_{e}^{2}}{\Phi_{1}^{2}}-\frac{k(t) v_{r} y_{e}^{2}}{\Phi_{1}^{2}}-k_{2} \frac{\bar{\theta}_{e}^{2}}{\Phi_{3}^{2}}+\frac{y_{e} g_{2}}{\Phi_{1}}-\tilde{v}\left(\frac{x_{e}}{\Phi_{1}}-\frac{k(t) x_{e} y_{e} \bar{\theta}_{e}}{\Phi_{1}^{2} \Phi_{2}}\right)-\tilde{\omega} \frac{\bar{\theta}_{e}}{\Phi_{3}}\left(1-\frac{k(t) x_{e}}{\Phi_{1}}\right)
$$

So far, the kinematic controller of the WCR has been derived. Next, the output of the kinematics controller is used as the auxiliary velocity input of the dynamics controller.

\section{Dynamic Analysis of the WCR}

\subsection{Analysis of Supporting Force of Wall-Climbing Robot}

The plane force diagram of the WCR in the robot coordinate system is shown in Fig. 3.

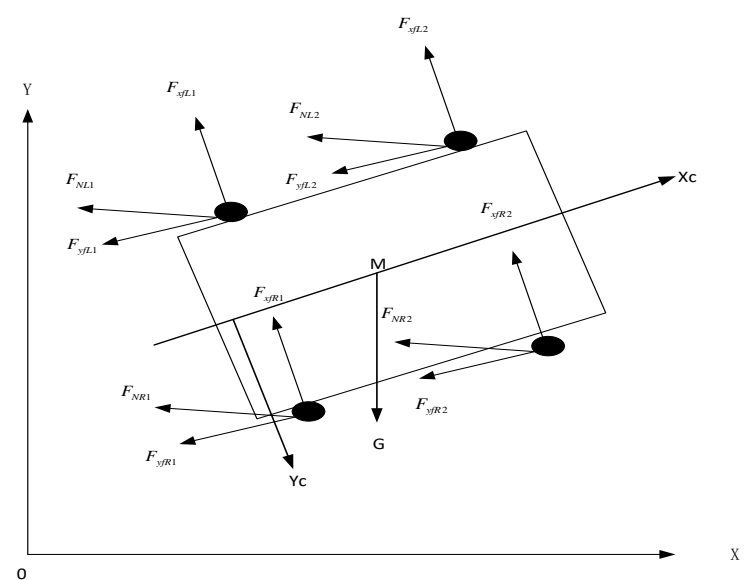

Fig. 3. Four-wheel drive mobile robot force diagram.

When the robot is in a state of static adsorption, the supporting force of the driving wheel on the wall satisfies the following relations,

$$
\left\{\begin{array}{c}
F_{N L 1}+F_{N R 1}+F_{N L 2}+F_{N R 2}=F_{P} \\
\left(F_{N L 2}+F_{N R 2}\right) d-\left(F_{N L 1}+F_{N R 1}\right) d+G h_{G} \sin \theta=0 \\
\left(F_{N L 2}+F_{N L 1}\right) b-\left(F_{N R 2}+F_{N R 1}\right) b+G h_{G} \cos \theta=0
\end{array}\right.
$$

where $F_{N L 1}$ is the supporting force on the wall of the rear left wheel, $F_{N R 1}$ is the supporting force on the wall of the rear right wheel, $F_{N L 2}$ is the supporting force on the wall of the front left wheel, $F_{N R 2}$ is the supporting force on the wall of the front right wheel, $F_{P}$ is the adsorption force of the robot, and $G$ is the overall weight of the robot.

The anti-inertia force $F_{x}$ in the horizontal direction and $F_{y}$ in the vertical direction are respectively defined as:

$$
\left\{\begin{array}{l}
F_{x}=-M \dot{x}_{c} \\
F_{y}=-M \dot{y}_{c}
\end{array}\right.
$$


where $\dot{x}_{c}$ and $\dot{y}_{c}$ are the horizontal and vertical velocity respectively.

(1) When $F_{P}$ acts alone, the supporting force of each driving wheel is

$$
F_{N L 1}{ }^{1}=F_{N R 1}^{1}=F_{N L 2}^{1}=F_{N R 2}{ }^{1}=\frac{F_{P}}{4}
$$

(2) When the combined forces of the two components of the gravity component $G \sin \theta$ in horizontal direction and the inertia force $F_{X}$ in horizontal direction simultaneously act, the supporting forces of the driving wheels are respectively,

$$
\left\{\begin{array}{c}
{F_{N L 1}^{2}}^{2}=F_{N R 1}^{2}=-\frac{h_{G}\left(F_{x}-G \sin \theta\right)}{4 d} \\
F_{N L 2}^{2}=F_{N R 2}^{2}=\frac{h_{G}\left(F_{x}-G \sin \theta\right)}{4 d}
\end{array}\right.
$$

(3) When the combined force of the gravity component $G \cos \theta$ in vertical direction and the inertia force $F_{y}$ in vertical direction, the supporting forces of the driving wheels are respectively,

$$
\left\{\begin{array}{c}
F_{N L 1}{ }^{3}=F_{N L 2}{ }^{3}=\frac{h_{G}\left(F_{y}-G \cos \theta\right)}{4 b} \\
F_{N R 1}^{3}=F_{N R 2}{ }^{3}=-\frac{h_{G}\left(F_{y}-G \cos \theta\right)}{4 d}
\end{array}\right.
$$

In the combined force of $F_{P}$, gravity $G$ and anti-inertial force $F_{x}$ and $F_{y}$, the supporting force of each driving wheel is

$$
\left\{\begin{array}{l}
F_{N L 1}=\frac{1}{4}\left[F_{P}+h_{G}\left(-\frac{F_{x}}{d}+\frac{F_{y}}{b}+\frac{G \sin \theta}{d}-\frac{G \cos \theta}{b}\right)\right] \\
F_{N R 1}=\frac{1}{4}\left[F_{P}+h_{G}\left(-\frac{F_{x}}{d}-\frac{F_{y}}{b}+\frac{G \sin \theta}{d}+\frac{G \cos \theta}{b}\right)\right] \\
F_{N L 2}=\frac{1}{4}\left[F_{P}+h_{G}\left(\frac{F_{x}}{d}+\frac{F_{y}}{b}-\frac{G \sin \theta}{d}-\frac{G \cos \theta}{b}\right)\right] \\
F_{N R 2}=\frac{1}{4}\left[F_{P}+h_{G}\left(\frac{F_{x}}{d}-\frac{F_{y}}{b}-\frac{G \sin \theta}{d}+\frac{G \cos \theta}{b}\right)\right]
\end{array}\right.
$$

Due to the uniqueness of the working environment, it is necessary to study the geometric constraint conditions of the wall-climbing robot to achieve a more complex trajectory movement. The steering mode of WCR is usually controlled by differential steering: the speed of the inside driving wheel is less than that of the outer driving wheel. Let $\rho$ be the robot steering radius, so the inner wheel steering angle is

$$
\alpha_{1}=\tan ^{-1} \frac{d}{\rho-b}
$$

The lateral steering angle is

$$
\alpha_{2}=\tan ^{-1} \frac{d}{\rho+b}
$$


The speed of no more than $2 \mathrm{~m} / \mathrm{s}$ is usually maintained to ensure the stability of the robot as it moves along the wall; the friction force and gravity are also necessary considerations. The friction force of the driving wheel can be divided into tangential friction force and transverse friction force.

The longitudinal friction force $F_{x f i}$ is

$$
F_{x f i}=\frac{\sigma}{r} F_{N i} \operatorname{sgn}\left(v_{x i}\right) \cos \alpha
$$

The transverse friction $F_{y f i}$ is

$$
\begin{aligned}
& F_{y f i}=\frac{\sigma}{r} F_{N i} \operatorname{sgn}\left(v_{y i}\right) \sin \alpha \\
& i=L 1 、 R 1 、 L 2 、 R 2
\end{aligned}
$$

where $\sigma$ is the driving wheel rolling friction coefficient.

Next, we derived the uncertain parameters of the dynamic model of wall-climbing robot.

\subsection{Dynamic Equation of Wall-Climbing Robot}

The robot maintains balanced forces on all sides while moving on the wall. The dynamic equation of the nonholonomic mobile robot system is based on a generalized coordinate system founded on the NewtonEuler equation [28]. The dynamics equations of this wheeled mobile robot are

$$
\left\{\begin{array}{c}
M \dot{v}_{x c}=F_{L}+F_{R}-G \sin \theta-\sum F_{x f i} \\
\operatorname{Mv}_{y c}=\sum \mathrm{F}_{\mathrm{yfi}}-\mathrm{G} \cos \theta \\
I \dot{w}=\left(F_{R}-F_{L}\right) b+\left(F_{x f L 1}+F_{x f L 2}-F_{x f R 1}-F_{x f R 2}\right) b+\left(F_{y f L 2}+F_{y f R 2}-F_{y f L 1}-F_{y f R 1}\right) d
\end{array}\right.
$$

In order to ensure the stability of the wall-climbing robot's movement on the wall surface, it is usually done at the low speed. Therefore, if $\dot{v}_{y c}=0$, the above formula can be rewritten as:

$$
\left\{\begin{array}{c}
M \dot{v}_{x c}=F_{L}+F_{R}-G \sin \theta-\sum F_{x f i} \\
\dot{M v}_{y c}=0 \\
I \dot{w}=\left(F_{R}-F_{L}\right) b+\left(F_{x f L 1}+F_{x f L 2}-F_{x f R 1}-F_{x f R 2}\right) b+\left(F_{y f L 2}+F_{y f R 2}-F_{y f L 1}-F_{y f R 1}\right) d
\end{array}\right.
$$

where

$$
\begin{gathered}
\sum F_{y f i}=\operatorname{sgn}(\dot{\theta}) \frac{\sigma}{2 r}\left\lfloor F_{P}\left(\sin \alpha_{1}+\sin \alpha_{2}\right)+\frac{h_{G} G \cos \theta\left(\sin \alpha_{1}-\sin \alpha_{2}\right)}{b}\right\rfloor \\
\sum F_{x f i}=\operatorname{sgn}(\dot{\theta}) \frac{\sigma}{2 r}\left\lfloor F_{P}\left(\cos \alpha_{1}+\cos \alpha_{2}\right)+\frac{h_{G} G \cos \theta\left(\cos \alpha_{1}-\cos \alpha_{2}\right)}{b}\right\rfloor \\
\left(F_{x f L 1}+F_{x f L 2}-F_{x f R 1}-F_{x f R 2}\right)=\operatorname{sgn}(\dot{\theta}) \frac{\sigma}{2 r}\left\lfloor F_{P}\left(\cos \alpha_{2}-\cos \alpha_{1}\right)-\frac{h_{G} G \cos \theta\left(\cos \alpha_{1}+\cos \alpha_{2}\right)}{b}\right\rfloor \\
\left(F_{y f L 2}+F_{y f R 2}-F_{y f L 1}-F_{y f R 1}\right)=\operatorname{sgn}(\dot{\theta}) \frac{\sigma}{2 r}\left\lfloor F_{P}\left(\sin \alpha_{1}+\sin \alpha_{2}\right)-\frac{h_{G} G \cos \theta\left(\sin \alpha_{1}-\sin \alpha_{2}\right)}{b}\right\rfloor
\end{gathered}
$$

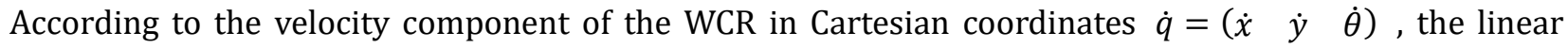
velocity component of the robot along its own coordinate system is obtained as: 


$$
\left\{\begin{array}{c}
v_{x c}=\dot{x} \cos \theta+\dot{y} \sin \theta \\
v_{y c}=-\dot{x} \sin \theta+\dot{y} \cos \theta
\end{array}\right.
$$

Take the derivative of the above equation,

$$
\left\{\begin{array}{c}
\dot{v}_{x c}=\ddot{x} \cos \theta-\dot{\theta} \dot{x} \sin \theta+\ddot{y} \sin \theta+\dot{\theta} \dot{y} \cos \theta \\
\dot{v}_{y c}=-\ddot{x} \sin \theta-\dot{\theta} \dot{x} \cos \theta+\ddot{y} \cos \theta-\dot{\theta} \dot{y} \sin \theta
\end{array}\right.
$$

If $A=M \dot{v}_{x c}, B=M \dot{v}_{y c}$, using $A$ and $B$ in formula (22):

$$
\left\{\begin{array}{l}
M \ddot{x}+M \dot{\theta} \dot{y}=A \cos \theta-B \sin \theta \\
M \ddot{y}-M \dot{\theta} \dot{x}=A \sin \theta+B \cos \theta
\end{array}\right.
$$

If $\lambda=-M(\dot{x} \cos \theta+\dot{y} \sin \theta) \dot{\theta}$ :

$$
\left\{\begin{array}{c}
M \dot{\theta} \dot{y}=\lambda \sin \theta \\
-M \dot{\theta} \dot{x}=-\lambda \cos \theta
\end{array}\right.
$$

Combining (23) with (24) to obtain the following equation,

$$
\left\{\begin{array}{c}
M \ddot{x}+\lambda \sin \sin \theta=\left(F_{L}+F_{R}\right) \cos \theta-G \sin \theta \cos \theta-\sum F_{x f i} \cos \theta \\
M \ddot{y}-\lambda \cos \cos \theta=\left(F_{L}+F_{R}\right) \sin \theta-G \sin \theta \sin \theta-\sum F_{y f i} \sin \theta \\
I \dot{W}=\left(F_{R}-F_{L}\right) b+\left(F_{x f L 1}+F_{x f L 2}-F_{x f R 1}-F_{x f R 2}\right) b+\left(F_{y f L 2}+F_{y f R 2}-F_{y f L 1}-F_{y f R 1}\right) d
\end{array}\right.
$$

Assume that the driving wheel torque vectors for the left and right sides of the wall-climbing robot are

$$
T=\left(\begin{array}{ll}
r F_{L} & r F_{R}
\end{array}\right)^{T}=\left(\begin{array}{ll}
\tau_{L} & \tau_{R}
\end{array}\right)^{T}
$$

Then (25) is expressed as a matrix,

$$
M(q) \ddot{q}+C(q) F_{P}+D(q) G=B(q) T+A^{T}(q) \lambda
$$

where

$$
\left.\begin{array}{c}
M(q)=\left(\begin{array}{ccc}
M & 0 & 0 \\
0 & M & 0 \\
0 & 0 & I
\end{array}\right), \quad B(q)=\frac{1}{r}\left(\begin{array}{cc}
\cos \theta & \cos \theta \\
\sin \theta & \sin \theta \\
-b & b
\end{array}\right), \\
\sin \cos \theta+\operatorname{sgn}(\dot{\theta}) \frac{\sigma}{2 r}\left[\frac{h_{G} G \cos \theta\left(\cos \alpha_{1}-\cos \alpha_{2}\right)}{b}\right] \cos \theta \\
\sin \theta \sin \theta+\operatorname{sgn}(\dot{\theta}) \frac{\sigma}{2 r}\left[\frac{h_{G} G \cos \theta\left(\sin \alpha_{1}-\sin \alpha_{2}\right)}{b}\right] \sin \theta \\
\operatorname{sgn}(\dot{\theta}) \frac{\sigma b}{2 r}\left[\frac{h_{G} G \cos \theta\left(\cos \alpha_{1}+\cos \alpha_{2}\right)}{b}\right]+\operatorname{sgn}(\dot{\theta}) \frac{\sigma d}{2 r}\left[\frac{h_{G} G \cos \theta\left(\sin \alpha_{1}-\sin \alpha_{2}\right)}{b}\right]
\end{array}\right)
$$




$$
C(q)=\left(\begin{array}{c}
\operatorname{sgn}(\dot{\theta}) \frac{\sigma}{2 r}\left(\cos \alpha_{1}+\cos \alpha_{2}\right) \cos \theta \\
\operatorname{sgn}(\dot{\theta}) \frac{\sigma}{2 r}\left(\sin \alpha_{1}+\sin \alpha_{2}\right) \sin \theta \\
-\operatorname{sgn}(\dot{\theta}) \frac{\sigma b}{2 r}\left(\cos \alpha_{2}-\cos \alpha_{1}\right)-\operatorname{sgn}(\dot{\theta}) \frac{\sigma d}{2 r}\left(\sin \alpha_{1}+\sin \alpha_{2}\right)
\end{array}\right)
$$

Taking equation (27) to left multiply $S^{T}(q)$, it can be seen $S^{T}(q) \cdot A^{T}(q)=0$ from the constraint condition, so equation (27) can be written as

$$
M(q)_{*} \ddot{q}+C(q)_{*} F_{p}+D(q)_{*} G+\tau_{d}=\tau_{*}
$$

where $\tau_{d}$ is an uncertain factor, $\tau_{d}=\left\{\begin{array}{c}M_{*}(q)=S^{T}(q) \cdot M(q) \\ C_{*}(q)=S^{T}(q) \cdot C(q) \\ D_{*}(q)=S^{T}(q) \cdot D(q) \\ \tau_{*}=S^{T}(q) \cdot B(q) T\end{array}\right.$.

\section{Trajectory Tracking Control Law Optimization Design}

The error relation between reference position $q_{r}$ and actual position $q$ of the wall-climbing robot can be expressed as,

$$
q_{e}=q-q_{r}
$$

Therefore, the challenge of WCR is to find the appropriate control law to satisfy the following equation,

$$
\lim _{t \rightarrow \infty} q_{e}=\lim _{t \rightarrow \infty} q-q_{r}=0
$$

In the previous section, the kinematic controller is based on back-stepping. The WCR motion trajectory is tracked using the sliding mode variable structure control method, using the exponential reaching law. The stability of the proposed control rate is proved by Lyapunov stability criterion. Next, a fuzzy system is established, taking sliding surface function as input and $\varepsilon$ as output based on the fuzzy basic function. Finally, according to the adaptive rules, the uncertain parameter $\tau_{d}$ of the mathematical model of the WCR is estimated and the stability of the adaptive control rate is proved.

\subsection{Sliding Mode Variable Structure Control}

The design of the sliding mode variable structure controller includes the switching function, which is designed to quickly reach the sliding surface, then the sliding mode with good dynamic quality is determined. The sliding mode variable structure control approach rate is designed according to the control requirements; it forms a clear sliding mode area to achieve the design purpose. The exponential reaching law is used in the paper. Before reaching the switching surface, the speed is faster and the approach time is shortened; when the switching surface is reached, the speed becomes very small, and chattering is weakened.

The sliding surface is chosen as,

$$
S=\dot{q}_{e}+c q_{e}
$$


where $c$ is the three-order diagonal matrix, $c=\operatorname{diag}\left(\begin{array}{lll}c_{1} & c_{2} & c_{3}\end{array}\right), c_{i}>0$. The reaching law [29] selected is

$$
\dot{S}=-k_{1} \operatorname{sgn}(S)-k_{2} S
$$

Here, $k_{1}=\operatorname{diag}\left(k_{11} \quad k_{12} \quad k_{13}\right), k_{2}=\operatorname{diag}\left(k_{21} \quad k_{22} \quad k_{23}\right), \quad k_{i j}>0, \quad \alpha$ is a constant between 0 and 1. Combining formula (29) with (31) to obtain the following equation,

$$
\ddot{q}=\ddot{q_{r}}-c \dot{q}_{e}+\dot{S}
$$

Using (33) in (28) obtains the sliding mode variable structure control law,

$$
\tau_{*}=M(q) \ddot{q}_{d}-M_{*}(q) c \dot{q}_{e}+M_{*}(q) \dot{S}+C_{*}(q) F_{p}+D_{*}(q) G+\tau_{d}
$$

The Lyapunov function [30] is chosen to analyze the stability of control law, setting the function as,

$$
V=\frac{1}{2} S^{T} M S
$$

Obviously, $V$ is a positive definite matrix, take the derivative of $V$ with respect to time,

$$
\dot{V}=\frac{1}{2}\left(\dot{S}^{T} M S+S^{T} \dot{M} S+S^{T} M \dot{S}\right)
$$

The combination of formulas (28), (31), (32), (36) results in the following equation,

$$
\dot{V}=S^{T}\left(-k_{2} S-k_{1}+\tau_{d}\right)=S^{T}\left(\tau_{d}-k_{1}\right)-S^{T} k_{2} S
$$

As $S^{T} k_{1} S$ is a positive definite matrix, according to (37), to make the $\dot{V}<0, \tau_{d} \leq k_{2}$ must be satisfied, guaranteeing that the sliding surface can be reached in a finite time; therefore, the control law is asymptotically stable.

\subsection{Fuzzy Control System}

Because the sliding surface is constantly switching, when $S \rightarrow 0, \quad k_{2} S \rightarrow 0$, but $\lim _{s \rightarrow 0} \varepsilon \operatorname{sgn}(S) \neq 0$; so that the system will further produce buffeting. To speed up the arrival time and reduce the chattering, the fuzzy system is established in the paper, taking $S$ and $\dot{S}$ as input and $\varepsilon$ as output to control the gain of $\varepsilon$. The fuzzy control algorithm based on fuzzy basis function is used to establish the fuzzy system between input and output, and the gain is adjusted by fuzzy rule.

Applying the IF-THEN rule, the fuzzy controller is constructed, and let the input and output linguistic variables of the fuzzy set be $\{$ NB,NS,ZO,PS,PB $\}$. Where NB means negative big, NS means negative small, ZO means zero, PS means positive small, PB means positive big. The fuzzy control rules can be expressed as: $F^{(J)}: I F s_{i}$ is $R_{S}{ }^{j} T H E N k_{i}$ is $C^{j}$, where $S_{i} \in S$, the fuzzy set is $R_{S}{ }^{j}, k_{i} \in k, C^{j}$ is the output of fuzzy rule $J$. The following fuzzy basis function [31] is chosen, 


$$
\xi_{i}(x)=\frac{\prod_{j}^{n} \mu_{R_{j}^{i}}\left(x_{j}\right)}{\sum_{i=1}^{m} \prod_{j=1}^{n} \mu_{R_{j}^{i}}\left(x_{j}\right)}
$$

where $x_{j}$ is any input variable, the membership function is $\mu_{R_{j}^{i}}\left(x_{j}\right), m$ and $n$ means fuzzy rule number.

Gauss membership function is chosen as the membership function for the fuzzy system. The Gauss type membership function is determined by the parameters $\sigma$ and $c . \sigma$ is positive and $c$ is used to determine the center of the curve. For $x$ is an independent variable, the representation of the Gauss type membership function is as follows,

$$
f(x, \sigma, c)=e^{-\frac{(x-c)^{2}}{2 \sigma^{2}}}
$$

According to the previous fuzzy basis function, the Gauss membership function is chosen,

$$
\left\{\begin{array}{l}
\mu_{N B}\left(x_{j}\right)=\exp \left\{-\left[\left(x_{j}+\pi / 6\right) /(\pi / 24)\right]^{2}\right\} \\
\mu_{N S}\left(x_{j}\right)=\exp \left\{-\left[\left(x_{j}+\pi / 12\right) /(\pi / 24)\right]^{2}\right\} \\
\mu_{Z O}\left(x_{j}\right)=\exp \left\{-\left[x_{j} /(\pi / 24)\right]^{2}\right\} \\
\mu_{P S}\left(x_{j}\right)=\exp \left\{-\left[\left(x_{j}-\pi / 12\right) /(\pi / 24)\right]^{2}\right\} \\
\mu_{P B}\left(x_{j}\right)=\exp \left\{-\left[\left(x_{j}-\pi / 6\right) /(\pi / 24)\right]^{2}\right\}
\end{array}\right.
$$

According to the fuzzy basis function and the gaussian membership function, the fuzzy control rules are shown in Table 1.

Table 1. Fuzzy Control Rule Table for Output Values $\varepsilon$

\begin{tabular}{llllll}
\hline & NB & NS & ZO & PS & PB \\
\hline NB & NB & NB & NS & NS & NB \\
NS & NB & NB & NS & NS & NS \\
ZO & PS & NS & ZO & PS & NS \\
PS & NB & NS & PS & PS & PS \\
PB & NB & NB & PS & PB & PB \\
\hline
\end{tabular}

In the application of the fuzzy inference system, using the product inference machine, the single valued fuzzy controller and the center average fuzzy controller, the fuzzy controller output is as follows,

$$
\varepsilon_{i}=\frac{\sum_{i=1}^{m}\left(\eta_{\varepsilon_{i}}^{i} \prod_{j=1}^{n} \mu_{R_{j}^{i}}\left(S_{j}\right)\right)}{\sum_{i=1}^{m} \prod_{j=1}^{n} \mu_{R_{j}^{i}}\left(S_{j}\right)}=\Lambda_{\varepsilon_{i}}^{T} \xi_{\varepsilon_{i}}\left(S_{i}\right)
$$

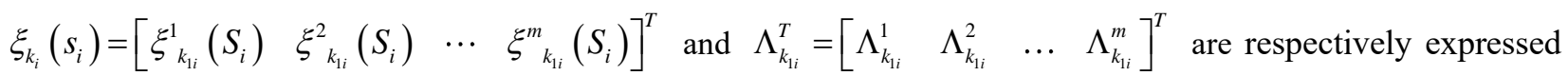
as the height vector and the center vector of the $k_{l}$ membership function. 


\subsection{Adaptive Control System}

Because of the uncertainty of $\tau_{d}$ in the dynamic model of the wall-climbing robot, the adaptive control law is used to estimate the questionable parameters of the control system.

From the proof of the Lyapunov stability of the control law, the following equations [32] are chosen to approximate the uncertainty of the model.

$$
\varepsilon=\Lambda^{T} \xi(S)
$$

where,

$$
\left\{\begin{array}{c}
\xi(S)=\left[\begin{array}{llll}
\xi_{k_{11}} & \xi_{k_{12}} & \cdots & \xi_{k_{1 m}}
\end{array}\right]^{T} \\
\Lambda=\left[\begin{array}{llll}
\Lambda_{k_{11}} & \Lambda_{k_{12}} & \cdots & \Lambda_{k_{1 m}}
\end{array}\right]
\end{array}\right.
$$

Let $\eta$ be the arbitrary positive real number. According to the approximate principle, the uncertain disturbance of the wall-climbing robot must satisfy the following inequality,

$$
\left\|\tau_{d}-\hat{\Lambda}^{T} \xi(S)\right\| \leq \eta \leq \lambda_{i}\|S\|
$$

where $\hat{\Lambda}$ is the estimated value of $\Lambda, \lambda$ is an integer between 0 and $1, i=1,2,3$.

Then the $\Lambda_{e}$ error of $\Lambda$ can be expressed as,

$$
\Lambda_{e}=\Lambda-\hat{\Lambda}
$$

As per the trajectory tracking control law of the wall-climbing robot, the adaptive law can be designed as,

$$
\dot{\Lambda}_{e}=S^{T} \xi(S)
$$

The Lyapunov function is chosen to carry out the stability analysis of the (46),

$$
V=\frac{1}{2} S^{T} M S+\frac{1}{2} \Lambda_{e}^{T} \Lambda_{e}
$$

Take the differential of (47) can be obtained

$$
V=\frac{1}{2}\left(\dot{S}^{T} M S+S^{T} \dot{M} S+S^{T} M \dot{S}\right)+\frac{1}{2}\left(\dot{\Lambda}_{e}^{T} \Lambda_{e}+\Lambda_{e}^{T} \dot{\Lambda}_{e}\right)
$$

Exploiting (37) in (48) obtains

$$
\dot{V}=S^{T}\left(\tau_{d}-k_{2}\right)-S^{T} k_{1} S+\Lambda_{e}^{T} \dot{\Lambda}_{e}
$$

Combining equation (42), (43), (45), (46), (49), then,

$$
\dot{V}=s^{T}\left[\tau_{d}-\hat{\Lambda}^{T} \xi(S)\right]-S^{T} k_{1} S
$$


Following the inequality (44), the equation (50) can be changed into the form of inequality,

$$
\dot{V} \leq S^{T}\left(\lambda-k_{1}\right) S \leq \sum_{i=1}^{3}\left(\lambda_{i}-k_{1 i}\right) S_{i}^{2}
$$

It can be seen from equation (51), if $k_{1 i}>\lambda_{i}$, so $\dot{V}$ is constantly less than zero. Meanwhile, when $S=0$, $\dot{V}=0$, then,

$$
\lim _{t \rightarrow \infty} S=\lim _{t \rightarrow \infty}\left[\left(\dot{q}-\dot{q}_{r}\right)+c\left(q-q_{r}\right)\right]=0
$$

Due to $\mathrm{c}$ is a positive definite matrix, so

$$
\lim _{t \rightarrow \infty} q=\lim _{t \rightarrow \infty} q_{r}, \lim _{t \rightarrow \infty} \dot{q}=\lim _{t \rightarrow \infty} \dot{q}_{r}
$$

The formula (53) proves the asymptotic stability of the designed trajectory tracking control law and verifies its feasibility.

The design of sliding mode fuzzy adaptive controller based on back-stepping of WCR trajectory tracking system is completed.

\section{Trajectory Simulation}

In this section, we verify the effectiveness and stability of the proposed control law through experiment. The mathematical model of a four-wheel drive wall-climbing robot is described by equation (10). The main parameters of the four-wheel-driven wall-climbing robot: $M=4.8 \mathrm{~kg}, J=3 \mathrm{~kg} . \mathrm{m}^{2}, d=0.1 \mathrm{~mm}, b=0.25 \mathrm{~m}, p=0.2 \mathrm{~m}$, $\mu_{s}=0.05, F_{p}=30 \mathrm{~N}, \delta=0.015, h_{G}=0.05, r=0.1 \mathrm{~m}$. Sliding mode controller: $k_{21}=0.01, k_{22}=0.02, k_{23}=0.03, \alpha=0.5$.

Scenario 1: WCR tracks the straight line trajectory, the reference trajectory linear equation is: $x_{r}(t)=t$, $y_{r}(t)=t, \theta_{r}(t)=\pi / 4$, the initial pose of the reference trajectory is $x_{r}(0)=0, y_{r}(0)=0, \theta_{r}(0)=0$. The initial position of the WCR is $x(0)=1.2, x(0)=1.2, y(0)=-2, \theta(0)=\pi / 2, v_{r}=0.3 \mathrm{~m} / \mathrm{s}, \omega=0 \mathrm{rad} / \mathrm{s}$. The simulation results are as follows,

Fig. 4 shows that the proposed BFASMC converges from the initial position to the reference line trajectory in 4 seconds, and the tracking error $\left(\begin{array}{lll}x_{e} & y_{e} & \theta_{e}\end{array}\right)$ converges to zero. The torque $(\tau)$ is also achieved to meet the requirements.

Scenario 2: WCR tracks the circular trajectory, the circular trajectory equation is $x_{r}(t)=4 v_{r} \sin \left(\omega_{r} t\right)$, $y_{r}(t)=4 v_{r} \sin \left(\omega_{r} t\right), \theta_{r}(t)=\omega_{r} t$. The initial pose of the reference trajectory is $x_{r}(0)=1, y_{r}(0)=-0.4$, $\theta_{r}(0)=\pi / 3$. The initial position of the WCR is $x(0)=0.5, y(0)=-0.5, \theta(0)=\pi / 4, v_{r}=0.3 \mathrm{~m} / \mathrm{s}, \omega=0.25$ $\mathrm{rad} / \mathrm{s}$. The simulation results are as follows,

Fig. 5 shows that the proposed BFASMC converges from the initial position to the reference circular trajectory in 3 seconds, and the tracking error $\left(\begin{array}{lll}x_{e} & y_{e} & \theta_{e}\end{array}\right)$ converges to zero. The torque $(\tau)$ is also achieved to meet the requirements. 


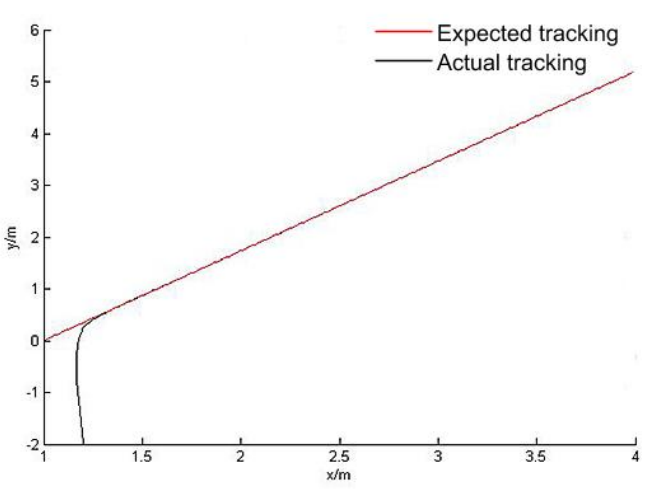

(a) BFASMC trajectory of the straight line

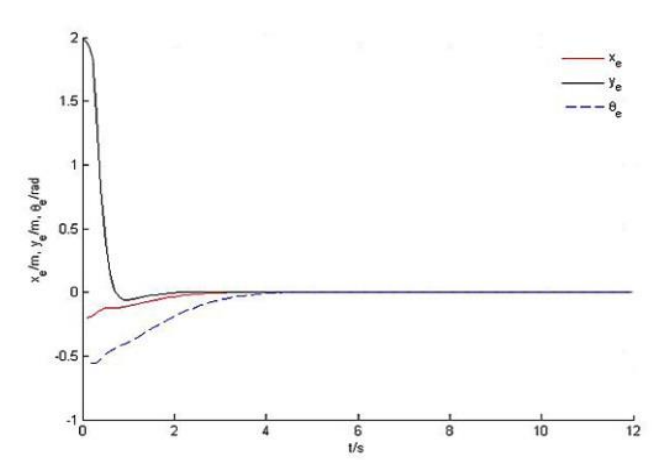

(c) BFASMC error of tracking straight line

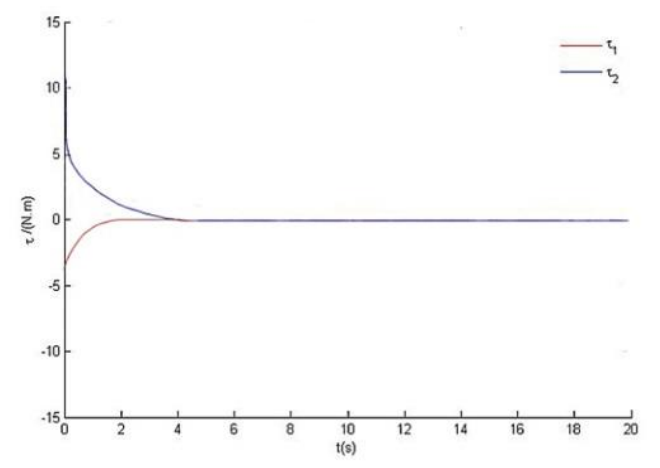

(e) BFASMC torque of tracking straight line Fig. 4. simulation curves of the straight line trajectory of WCR.

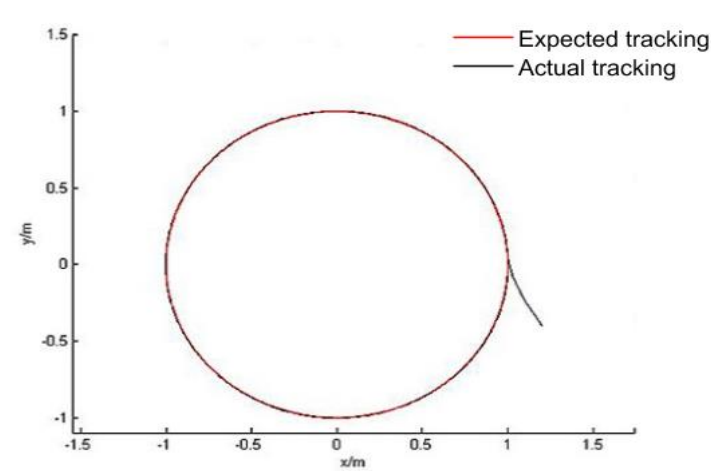

(a) BFASMC trajectory of the circular

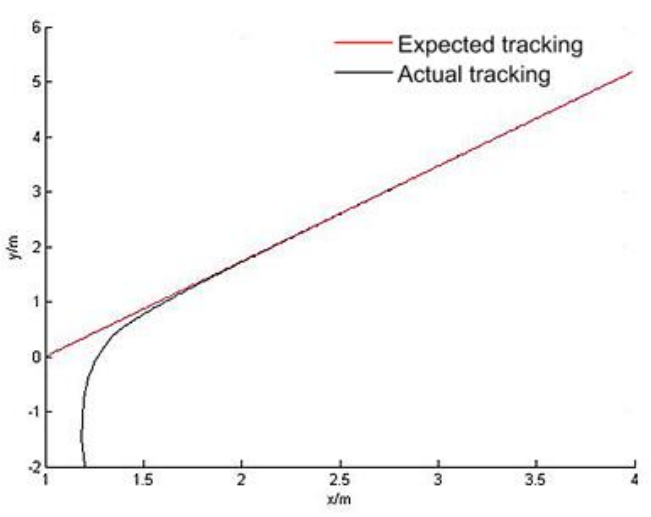

(b) BASMC trajectory of the straight line

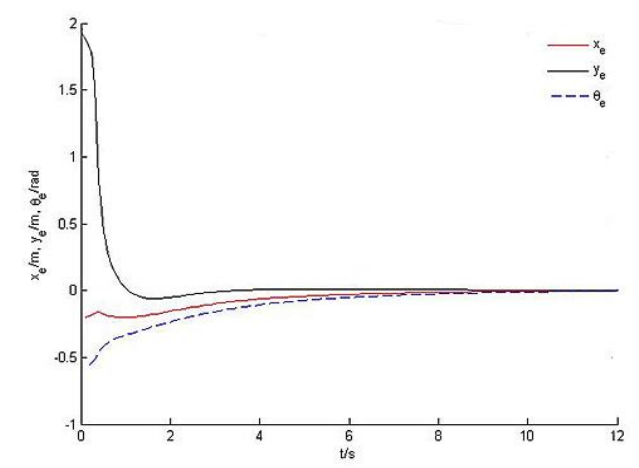

(d) BASMC error of tracking straight line

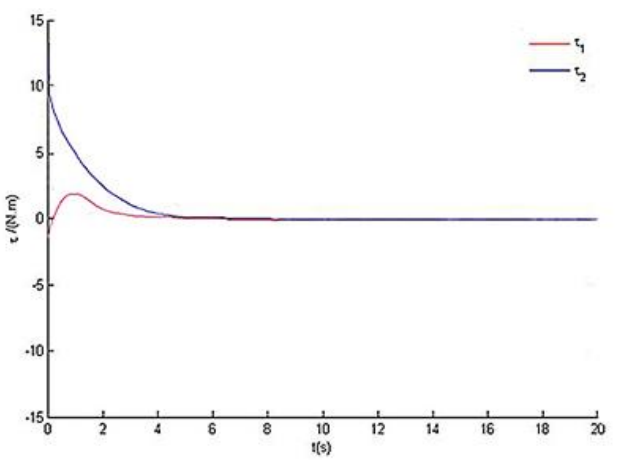
(f) BASMC torque of tracking straight line

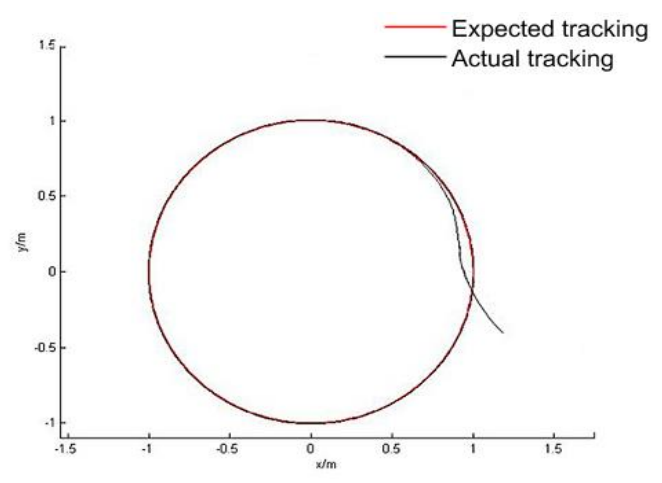

(b) BASMC trajectory of the circular 


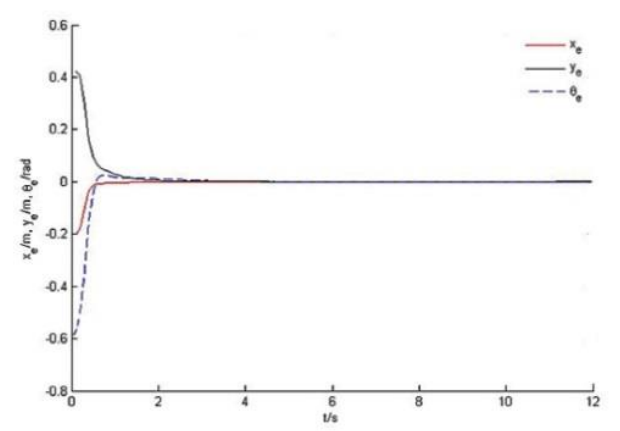

(c) BFASMC error of tracking the circular

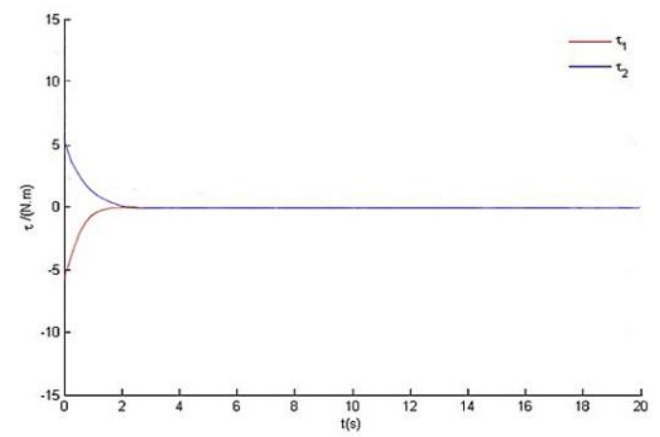

(e) BFASMC torque of tracking the circular

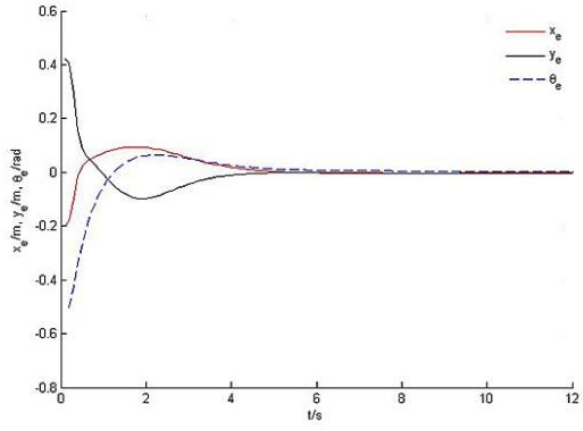

(d) BASMC error of tracking the circular

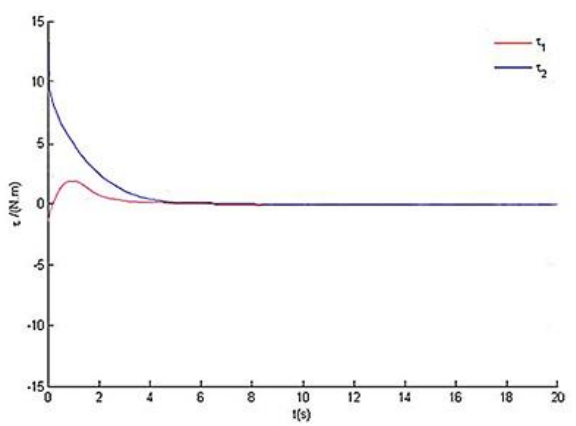

(f) BASMC torque of tracking the circular

Fig. 5. Simulation curves of the circular trajectory of WCR.

Table 2. The Pose Error Convergence Time Dynamic Controllers

\begin{tabular}{ccc}
\hline $\begin{array}{c}\text { control } \\
\text { method }\end{array}$ & $\begin{array}{c}\text { Pose error convergence time (s) } \\
\text { straight line }\end{array}$ & $\begin{array}{c}\text { Pose error convergence time(s) } \\
\text { circle }\end{array}$ \\
\hline The BASMC & 7.2 & 6 \\
The BFASMC & 4 & 3 \\
\hline
\end{tabular}

The result shows that the BFASMC achieves better performance compared to BASMC in tracking the straight line and circular trajectory. Compared with the BASMC, the pose error convergence time of the proposed BFASMC in tracking the straight line and circular trajectory are respectively shortened by 3.2 seconds and by 3 seconds in Table 2 .

\section{Conclusions}

In this paper, we proposed a novel trajectory tracking system of WCR based BFASMC. To further reduce the steady-state error of the control law and the number of switching times of the sliding mode surface, we firstly explored that the output of the kinematics controller based back-stepping is considered as the auxiliary velocity input of the dynamics controller. Furthermore, adding fuzzy control algorithm structure based on fuzzy basis function was feasible to efficiently improve pose error convergence speed and trajectory tracking performance. Then an adaptive control method is added to adjust the uncertainty of the system. The experimental results show that the proposed tracking algorithm has better tracking effect and stability than the state-of-the-art methods.

\section{Acknowledgement}

This work was supported in part by the Sichuan Science and Technology Department Application 
Foundation Project 2019YJ0311, in part by the Open Fund of the Key Laboratory of Oil and Gas Equipment, Ministry of Education under Grant OGE201702-06, in part by the State Administration of National Security under Grant sichuan-0006-2018AQ and in part by Scientific Research Starting Project of Southwest Petroleum University under Grant 2015QHZ010.

\section{Reference}

[1] Ahmad, 0., Ullah, I., \& Iqbal, J. (2014). A multi-robot educational and research framework. Int. J. Acad. Res., 6(2), 217-222.

[2] Fabien, T., Wolfgang, F., Gilles, C., Rol, S., Rol, M., \& Francesco, M. (2007). Compact magnetic wheeled robot with high mobility for inspecting complex shaped pipe structures. Proceedings of Ieee/rsj International Conference on Intelligent Robots and Systems: Vol. 26 (pp. 261-266). San Diego, California, USA: Sheraton Hotel and Marina.

[3] Tummala, R. L., Mukherjee, R., Xi, N., Aslam, D., Dulimarta, H., \& Xiao, J. (2002). Climbing the walls. IEEE Robotics \& Automation Magazine, 9(4), 10-19.

[4] Fang, Z., Zhi, Z., Jun, L., \& Jian, W. (2008). Feedback linearization and continuous sliding mode control for a quadrotor UAV. Proceedings of the 27th Chinese Control Conference (pp. 349-353). Kunming, China.

[5] Pathak, K., Franch, J., \& Agrawal, S. K.. Velocity and position control of a wheeled inverted pendulum by partial feedback linearization. IEEE Transactions on Robotics, 21(3), 505-513.

[6] Tee, K. P., Ren, B., \& Ge, S. S. (2011). Control of nonlinear systems with time-varying output constraints. Automatica, 47(11), 2511-2516.

[7] Gradetsky, V. G., \& Knyazkov, M. M. (2014). Wall climbing robots: Mechanics, control and adaptation to environment. Proceedings of International Conference on Robotics in Alpe-Adria-Danube Region (pp. 1-7).

[8] Lizarralde, F., Leite, A. C., Hsu, L., \& Costa, R. R. (2013). Adaptive visual servoing scheme free of image velocity measurement for uncertain robot manipulators. Automatica, 49(5), 1304-1309.

[9] Iqbal, J., Islam, R. U., \& Khan, H. (2012). Modeling and analysis of a 6 DOF robotic arm manipulator. Can. J. Electr. Electron. Eng., 3(6), 300-306.

[10] Dutta, S., Patchaikani, P. K., \& Behera, L. (2016). Near-optimal controller for nonlinear continuous-time systems with unknown dynamics using policy iteration. IEEE Trans. Neural Netw. Learn. Syst., 27(7), 1537-1549.

[11] Evans, J., \& Nahon, M. (2004). Dynamics modeling and performance evaluation of an autonomous underwater vehicle. Ocean Engineering, 31, 1835-1858.

[12] Yan, Y., Xu, J., \& Wiercigroch, M. (2017). Basins of attraction of the bistable region of time-delayed cutting dynamics. Phys. Rev. E, Stat. Phys. Plasmas Fluids Relat. Interdiscip. Top., 96(3), 032205.

[13] Asif, M., Khan, M. J., \& Cai, N. (2014). Adaptive sliding mode dynamic controller with integrator in the loop for nonholonomic wheeled mobile robot trajectory tracking. International Journal of Control, 87(5), 964-975.

[14] Liang, R., Liu, R., \& Yang, C. (2013). Design, modeling and analysis of a climbing robot used for inspection of glass curtain walls. Proceedings of 2013 IEEE International Conference on Robotics and Biomimetics (ROBIO) (pp. 402-407).

[15] Polyakov, A., \& Fridman, L. (2014). Stability notions and lyapunov functions for sliding mode control systems. Journal of the Franklin Institute, 351(4), 1831-1865.

[16] Iván, G., Sergio, S., \& Rodríguez-Mata, A. E. (2017). Filiberto M. P., Ricardo L., Rogelio L.: Enhanced robust altitude controller via integral sliding modes approach for a quad-rotor aircraft: Simulations and realtime results. Journal of Intelligent \& Robotic Systems, 88, 313-327.

[17] Zou, A. M., Kumar, K. D., Hou, Z. G., \& Liu, X. (2011). Finite-time attitude tracking control for spacecraft 
using terminal sliding mode and Chebyshev neural networks. IEEE Trans. Syst., Man, Cybern. B, Cybern., 41(4), 950-963, 2011.

[18] Fallaha, C. J., Saad, M., Kanaan, H. Y., \& Al-Haddad, K. (2011). Sliding-mode robot control with exponential reaching law. IEEE Trans. Ind. Electron, 58(2), 600-610.

[19] Mohan, S., \& Mohanta, J. K. (2018). Dual integral sliding mode control loop for mechanical error correction in trajectory-tracking of a planar 3-PRP parallel manipulator. Journal of Intelligent \& Robotic Systems, 89, 371-385.

[20] Keshtkar, S., Poznyak, A. S., \& Hernandez, E. (2017). Adaptive sliding-mode controller based on the "Super-Twist" state observer for control of the Stewart platform. Automation and Remote Control, 78(7), 1218-1233.

[21] Rossomando, F. G., Soria, C., \& Carelli, R. (2014). Sliding mode neuro adaptive control in trajectory tracking for mobile robots. Journal of Intelligent \& Robotic Systems, 74(3), 931-944.

[22] Asif, M., Khan, M. J., \& Cai, N. (2014). Adaptive sliding mode dynamic controller with integrator in the loop for nonholonomic wheeled mobile robot trajectory tracking. International Journal of Control, 87(5), 964-975.

[23] Fang, J., Zhong, W., Li, C., Zhao, Z., \& Wang, M. Y. (2019). Fuzzy adaptive multi-mode sliding mode control for precision linear stage based on floating stator. Review of Scientific Instruments, 90(1), 015103.

[24] Gong, X., Hou, Z. C., \& Zhao, C. J. (2012). Adaptive backstepping sliding mode trajectory tracking control for a quad-rotor. International Journal of Automation and Computing, 9(5), 555-560.

[25] Rajendran, S., \& Jena, D. (2014). Backstepping sliding mode control for variable speed wind turbine. Proceeding of India Conference. IEEE.

[26] Mohareri, O., Dhaouadi, R., \& Rad, A. B. (2012). Indirect adaptive tracking control of a nonholonomic mobile robot via neural networks. Neurocomputing, 88(7), 54-66.

[27] Xu, W., Jiang, J., Zhen, Z., \& Li, X. (2008). Near space vehicle control based on back-stepping robust adaptive dynamic plane. Electro-Optic and Control, 25(11), 15-20.

[28] He, Z., Song, X., \& Xue, D. (2016). Comments to the "Closed-form dynamic equations of the general Stewart platform through the Newton-Euler approach" and "A Newton-Euler formulation for the inverse dynamics of the Stewart platform manipulator". Mechanism and Machine Theory, 102, 229-231.

[29] Liu, H., Wang, H., \& Sun, J. (2019). Attitude control for QTR using exponential nonsingular terminal sliding mode control. Journal of Systems Engineering and Electronics, 30(01), 191-200.

[30] Ren, B., Ge, S. S., Tee, K. P., \& Lee, T. H. (2010). Adaptive neural control for output feedback nonlinear systems using a barrier Lyapunov function. IEEE Trans. Neural Netw., 21(8), 1339-1345.

[31] Mai-Cao, L., \& Tran-Cong, T. (2014). A meshless numerical approach based on integrated radial basis functions and level set method for interfacial flows. Applied Mathematical Modelling, 38(23), 5743-5766.

[32] Qu, Q., Zhang, H., \& Feng, T. (2017). Decentralized adaptive tracking control scheme for nonlinear largescale interconnected systems via adaptive dynamic programming. Neurocomputing, 225, 1-10.

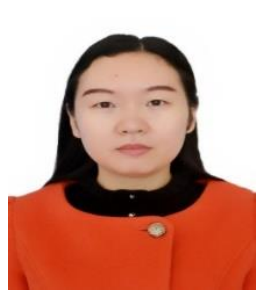

Xin Lai was born in Nanchong, China. Currently, she is an associate professor at the Southwest Petroleum University. During her integrated experience several projects, prototypes and publications are conducted, specialized in the following research areas: robotics, deep learning, mechatronics system design, sensor fusion, fault diagnostics, intelligent control, water quality monitoring and wastewater treatment. She is an IEEE member. 


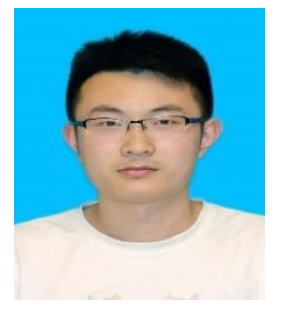

Pengli Liu was born in Neijiang, China. He received a bachelor's degree in electromechanical engineering from Southwest Petroleum University in 2017. He is currently studying for a master's degree at Southwest Petroleum University. His current research direction is in the field of intelligent robots.

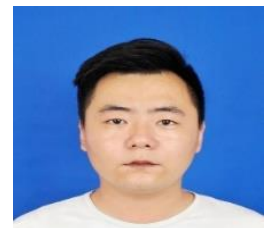

Yang Lu was born in Deyang, China. He received a bachelor's degree and master's degree in the school of electromechanical engineering from Southwest Petroleum University in 2015 and 2018. He is currently working in Deyang College of Urban Rail Transit.

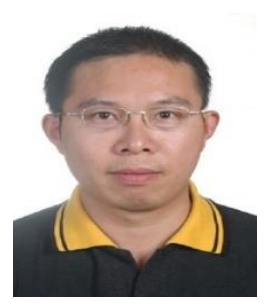

Chunpu Wang was born in Renshou, China. Now he is at Chuanqing Geological Exploration and Development Research Institute. He is an engineer research and development of oil and gas equipment. His current research is intelligent control of digital oil field. 